\title{
Atención psicológica especializada a mujeres víctimas y/o sobrevivientes de violencia en la Unidad Delegación de la Mujer del Instituto Nacional de las Mujeres ${ }^{1}$
}

\author{
Specialized psychological care for women victims and / or survivors \\ of violence in the "Unidad Delegación de la Mujer del Instituto \\ Nacional de las Mujeres
}

Ariela Leandro Espinoza ${ }^{2}$

\begin{abstract}
RESUMEN
La violencia contra las mujeres es una violación de los derechos humanos con gran impacto en la salud pública. En compromiso con convenciones internacionales de derechos humanos de las mujeres, en Costa Rica se creó el Instituto Nacional de las Mujeres, al cual pertenece la Unidad Delegación de la Mujer como una unidad de atención especializada a mujeres víctimas y/o sobrevivientes de violencia. Sin embargo, este cuenta con un personal reducido ante la alta demanda de atención; por lo que se planteó como objetivo proporcionar mayor recurso humano a este servicio a través de la atención psicológica especializada, desde un enfoque basado en los derechos humanos y género sensitivo. Las labores que se realizaron fueron la atención psicológica individual, intervenciones grupales breves a las mujeres que se encontraban esperando en la sala de espera, y la atención terapéutica grupal. Además de la atención en rol (la cual es la valoración inicial de las mujeres que solicitaban atención por primera vez en el servicio), la planificación e implementación de talleres psicoeducativos, y la participación en una entrevista de una víctima de trata de personas.

Palabras clave: violencia en contra de las mujeres, enfoque género sensitivo, enfoque basado en los derechos humanos, atención psicológica.
\end{abstract}

\footnotetext{
ABSTRACT

Violence against women is a violation of human rights with a major impact on public health. In commitment to international conventions on women's human rights, in Costa Rica the Instituto Nacional de las Mujeres was created, to which the Unidad Delegación de la Mujer belongs as specialized care unit for women victims and/or survivors of violence. However, it has a reduced staff due to the high demand for care; therefore, the objective was to provide greater human resources to this service through specialized psychological care, from a human rights-based and gender sensitive approach. The work that was carried out was individual psychological care, brief group interventions to women waiting in the waiting room, and group therapeutic care. In addition to role care (which is the initial assessment of women seeking first time care in the service), planning and implementing psychoeducational workshops, and participating in an interview with a victim of human trafficking.

Key Words: violence against women, sensitive gender approach, human rights-based approach, psychological care.

${ }^{1}$ Este artículo es producto de la práctica dirigida para optar por el grado de Licenciatura en Psicología de la autora, titulada "Atención terapéutica individual y grupal e intervenciones grupales breves a mujeres víctimas y/o sobrevivientes de violencia por la pareja en la Delegación de la Mujer del Instituto Nacional de las Mujeres", y como parte de la recomendación de publicación del Tribunal Examinar en la defensa pública virtual de ese trabajo final de graduación.

DOI: $10.15517 /$ WL.V16I2.47997

${ }^{2}$ Universidad de Costa Rica. Licenciada en Psicología. San José, Costa Rica. Correo electrónico: arile28@gmail.com ORCID: https://orcid.org/0000-0003-2574-3583

Recepción: 20/2/2021 Aceptación: 10/6/2021
} 


\section{Introducción}

La violencia contra las mujeres es una problemática con un gran impacto en la salud pública y es una violación de los derechos humanos (OMS 2013). Asimismo, esta ocasiona consecuencias en detrimento de la salud de las mujeres, al provocar lesiones físicas (desde leves, hasta muy graves, que pueden provocar la muerte), trastornos por estrés postraumático, depresión, suicidio, ansiedad, embarazos no deseados, abortos, infecciones de transmisión sexual, entre otros (OPS y CDC 2014). La violencia por parte de la pareja es la violencia más frecuentemente experimentada por las mujeres, teniendo a nivel mundial una prevalencia del $30 \%$; además, de ser sus parejas responsables del 38\% de las muertes de mujeres (OMS 2013). Similarmente, esta es una problemática extendida en toda América Latina y el Caribe (OPS y CDC 2014).

En Costa Rica, según la única Encuesta Nacional de Violencia contra las Mujeres (realizada en el 2003), un 57,7\% de las mujeres entrevistadas reportó haber sufrido al menos un incidente de violencia física o sexual desde sus 16 años en adelante. En más del 60\% de los casos reportados quienes perpetraron de la violencia física fueron principalmente sus parejas (esposos, compañeros o novios), y también quienes forzaron a mantener relaciones sexuales a un $78,1 \%$ de las mujeres (Sagot y Guzmán 2004). Sobre la Ley contra Violencia Doméstica (Ley 7586/1996), para el 2019 en el 80\% de las medidas de protección solicitadas las víctimas eran mujeres (Observatorio de Violencia de Género contra las Mujeres y Acceso a la Justicia 2020a). De acuerdo con la Ley de Penalización de la Violencia Contra las Mujeres (Ley 8589/2007), en el 2018 se presentaron 20.130 denuncias y para el 2019 se registraron 21.122 denuncias por delitos relacionados con violencia física, psicológica, sexual y patrimonial en relaciones de matrimonio (Observatorio de Violencia de Género contra las Mujeres y Acceso a la Justicia 2020b). En cuanto a los femicidios, durante el 2019, se contabilizaban 16 de un total de 49 muertes violentas de mujeres, y para el año 2020 se registraron 12 femicidios de un total de 61 homicidios en contra de mujeres (Observatorio de Violencia de Género contra las Mujeres y Acceso a la Justicia 2020c).

Ante esta problemática, en el país se ratificaron convenciones internacionales de derechos humanos de las mujeres, como la Convención sobre la Eliminación de todas las formas de Discriminación contra la Mujer (Ley 6968/1984) y la Convención Interamericana 


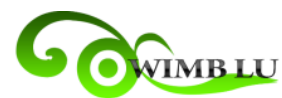

Wimblu, Rev. Estud. de Psicología UCR, 16(2) 2021 (Julio-Diciembre): 67-92 /ISSN: 1659-2107

para Prevenir, Sancionar y Erradicar la Violencia contra las Mujeres (Ley 7499/1995), lo que compromete al Estado costarricense a condenar la discriminación y la violencia en contra de las mujeres, a velar por sus derechos, y a atender a las mujeres objeto de esa violencia a través de servicios especializados y programas de rehabilitación y capacitación.

Como respuesta a estos compromisos, y aunado a las demandas y reivindicaciones del movimiento de mujeres y feministas (Sagot 2008), en el país se ha creado legislación para proteger a las mujeres y penalizar la violencia contra las mismas. De esta forma, en 1998 se creó el Instituto Nacional de las Mujeres (INAMU) con la misión de promover y proteger los derechos de las mujeres y coordinar la política nacional para la igualdad y la equidad de género (INAMU 2010). La Unidad Delegación de la Mujer del INAMU ${ }^{3}$ es una unidad de atención del Departamento de Violencia de Género del INAMU, la cual ofrece acompañamiento especializado a mujeres víctimas y/o sobrevivientes de violencia intrafamiliar, sexual y de hostigamiento sexual en el empleo o en la docencia del Gran Área Metropolitana (Morales y Aguilar 2016). En la Unidad Delegación, para el año 2018 se registraron 8.124 atenciones individuales en total, de las cuales 2.819 (alrededor del 35\%) eran consultas al servicio de psicología; mientras que para el 2019 se reportaron 3.141 atenciones psicológicas de un total de 8.264 atenciones individuales, lo que equivale al 39\% aproximadamente (INAMU 2020).

Sin embargo, aunque la Unidad Delegación cuenta con un modelo de intervención integral, apropiado y de calidad, y con un personal altamente capacitado y con amplia experiencia, el mismo es “(...) limitado para la alta demanda de atención, así como la complejización y aumento de algunos temas como por ejemplo, trata [de mujeres], acoso sexual y laboral, delitos cibernéticos, entre otros" (Morales y Aguilar 2016, 54), por lo que a las mujeres en situación de mayor amenaza se les atendía inmediatamente, mientras que aquellas en menor riesgo se les brindaba una cita con disponibilidad promedio de un mes (Morales y Aguilar 2016). Además, para inicios del mes de enero del 2019 solamente se contaba con 3 plazas para el área de psicología y las citas de atención se estaban brindando con un plazo de espera de 2 meses aproximadamente, de acuerdo con la Jefatura de la Unidad Delegación, Mag. Erika Rojas Calderón (comunicación personal, 30 de enero del 2019).

\footnotetext{
${ }^{3}$ En adelante este artículo se referirá a esta como Unidad Delegación.
} 


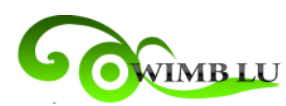

Wimblu, Rev. Estud. de Psicología UCR, 16(2) 2021 (Julio-Diciembre): 67-92 /ISSN: 1659-2107

Lo expuesto anteriormente indujo a considerar la urgencia de brindar atención especializada y garante de sus derechos humanos a mujeres expuestas a violencia, así como a cuestionar sobre la limitación de personal para atender esta problemática; por lo que se planteó como objetivo proporcionar mayor recurso humano a la Unidad Delegación a través de la atención psicológica especializada desde un enfoque basado en los derechos humanos y género sensitivo.

\section{Enfoques teórico-conceptuales}

En primer lugar, con respecto al enfoque basado en derechos humanos (EBDH) se fundamenta en los valores, principios y normas universales en los tratados de los derechos humanos, ligados a la dignidad humana y al desarrollo integral de las personas (García y Borja 2014). Además, el EBDH considera la irreductibilidad de los derechos de las mujeres y el deber de garantizar y proteger esos derechos por parte del Estado (INAMU 2017a), lo que implica que las personas prestatarias de servicios de atención deben de conocer y respetar los derechos de las mujeres víctimas y/o sobrevivientes de violencia, así como los principios éticos estipulados en instrumentos de derechos humanos (Organización Internacional para las Migraciones 2007). Además, deben tratarlas justa y respetuosamente, sin ningún tipo de discriminación, y considerando las múltiples formas de discriminación que puede estar enfrentando (OPS 2016).

En segundo lugar, se trabajó desde el enfoque género sensitivo, el cual considera que las interacciones sociales entre mujeres y hombres están permeadas por atribuciones y representaciones sociales y culturales aceptadas de acuerdo al género, por lo que cuestiona los estereotipos de género y plantea nuevas formas de socialización e interacción entre los seres humanos (Rodríguez, Robledo y Pedroso 2010). Además, para este es fundamental reconocer el impacto de la sociedad machista en la salud mental de las mujeres (Batres 2013), ya que el estatus de subordinación de las mujeres tiene efectos psicológicos directos en estas

y produce síntomas psicológicos (Batres 2009). Por lo anterior, desde el enfoque género sensitivo es central reconocer que la direccionalidad de esta violencia se vincula con el sistema patriarcal, en el que el solo hecho de ser mujer la coloca en posición de subordinación 


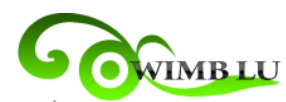

Wimblu, Rev. Estud. de Psicología UCR, 16(2) 2021 (Julio-Diciembre): 67-92 /ISSN: 1659-2107

y es factor de vulnerabilidad (INAMU 2017a). De esta forma, el trabajo con las mujeres debe de incluir un análisis de esas diferencias de poder con respeto a los hombres, con la finalidad de que lo recuperen de vuelta, validando sus percepciones y emociones, potenciando su capacidad de cuidarse y estimulando su vida independiente (Batres 2009 2013).

\section{Metodología}

\section{Participantes}

El número de participantes varió de acuerdo al tipo de atención brindada y, en el caso de algunas mujeres, fueron atendidas en varias de estas modalidades de atención.

En cuanto a la atención individual participaron 28 mujeres, cuyas edades oscilaron entre los 19 hasta los 56 años. Sobre sus nacionalidades, el 79\% (22 mujeres) eran costarricenses; 2 eran nicaragüenses (7\%); y una usuaria de cada uno de los siguientes países: República Dominicana, Honduras, El Salvador y Estados Unidos. Sobre el estado civil, 17 mujeres (61\%) estaban solteras; 7 mujeres (25\%) estaban casadas; 2 mujeres estaban divorciadas y 2 en unión libre. Sobre el nivel de escolaridad de las usuarias en atención individual, destaca que el $42 \%$ de las mujeres en esta modalidad de atención no terminaron sus estudios de primaria o secundaria. Con respecto a la ocupación, la mitad de ellas (14 usuarias) estaban desempleadas; 5 mujeres realizaban oficios técnicos; 2 eran dependientes de tiendas; 2 eran docentes; y una trabajaba en su negocio propio.

En la atención grupal la participación máxima fue de 15 mujeres, sus edades rondaron más frecuentemente entre los 20 hasta los 39 años. El estado civil de la mayoría de estas mujeres (72\%) eran solteras; un 17\% en unión libre; una usuaria era casada y otra divorciada. Sobre el nivel de escolaridad de las usuarias participantes, sobresale que el $62 \%$ no concluyeron sus estudios de primaria o secundaria, mientras que sólo un $8 \%$ continuó los estudios luego del colegio. En cuanto a su ocupación, el 70\% se encontraban desempleadas; el $24 \%$ realizaba oficios técnicos, y solo una mujer era dependiente en un negocio. 
En las intervenciones grupales breves en la sala de espera participaron 111 usuarias $^{4}$.

En cuanto a otras tareas solicitadas por la institución, en la atención en rol participaron 64 usuarias; de los talleres psicoeducativos formaron parte 79 mujeres; y solamente se realizó la entrevista con una usuaria víctima de trata de personas.

\section{Procedimiento}

Estrategias de intervención en la atención psicológica individual

El apoyo de primera línea a mujeres víctimas de violencia de pareja o agresión sexual es fundamental, ya que provee atención de urgencia centrándose en las necesidades de las mujeres, y porque esta puede ser la única atención a la que una mujer tenga oportunidad de acceder o necesite (OPS 2016). Esa atención de primer orden consta de 5 tareas: 1) atención al escuchar, 2) no juzgar y validar, 3) informarse sobre sus necesidades y preocupaciones, 4) mejorar la seguridad, y 5) apoyar, con información y otros servicios de salud, seguridad o apoyo social (OPS 2016).

De acuerdo con el Protocolo interinstitucional de intervención, valoración y administración del riesgo en situaciones de violencia contra las mujeres en relaciones de pareja (INAMU 2017b), la Unidad Delegación debe de actuar en primer lugar evaluando la situación de riesgo, lo que se realizó a través de la Escala de Valoración de riesgo de femicidio, el cual es un instrumento interno de este servicio que identifica el nivel de riesgo para la vida de las mujeres. Cuando se identifica riesgo alto de femicidio, la intervención debe ser inmediata para proteger a la víctima (INAMU 2017b). Luego se informa sobre las medidas de protección y la opción de denunciar los delitos, a través de los Juzgados específicos (de Violencia Doméstica, de Familia o Contravencional) o al Ministerio Público, respectivamente (INAMU 2017b). Además, se les brinda la opción del albergue para mujeres; si rechaza el traslado se define un plan de seguridad, el cual se elaboraba en conjunto con la mujer usuaria, tomando en consideración sus necesidades y realidades (INAMU 2017b). Adicionalmente, para las mujeres en mayor riesgo o presencia de extrema violencia,

\footnotetext{
${ }^{4}$ No se cuenta con datos demográficos de las usuarias participantes de las intervenciones en la sala de espera debido a lo breves que eran estas y a las características del grupo, tal y como se detalla más adelante.
} 
Wimblu, Rev. Estud. de Psicología UCR, 16(2) 2021 (Julio-Diciembre): 67-92 /ISSN: 1659-2107

se cuenta con un dispositivo denominado "Kit de emergencia o botón del pánico", el cual, cuando es activado por una mujer que se sienta en riesgo, emite una alarma para que la Policía se presente lo más pronto posible a su ubicación.

En cuanto a la intervención de segundo orden, se incluyó un seguimiento y monitoreo de las acciones que la usuaria llevó a cabo, según sus metas, objetivos y posibilidades, ya que este previene que la violencia se siga agudizando (Deza 2016). También, se trabajó la recuperación de las secuelas y el abordaje de problemas asociados a la violencia, ya que las consecuencias emocionales de esta problemática son profundas y requieren de una relación terapéutica construida con el tiempo y basada en la confianza (Ministerio de Desarrollo Social 2010).

Sobre el mecanismo de referencia y contra-referencia, deben de ser ágiles, efectivos y articulados, evitando la re-victimización de las mujeres, siguiendo lo señalado por la Política Nacional para la Atención y la Prevención de la violencia contra las mujeres de todas las edades 2017-2032 (INAMU 2017a).

\section{Estrategias de intervención en la atención psicológica grupal}

Sobre el proceso de selección de las mujeres para formar parte del grupo, las profesionales de Trabajo Social, Derecho y Psicología del servicio definieron, de acuerdo a su criterio técnico, cuáles de las mujeres que estaban atendiendo podían participar en la atención terapéutica grupal. Se consideraron como criterios de exclusión: mujeres en alto riesgo de femicidio; mujeres que recién entraron al servicio; mujeres con consumo problemático de sustancias (a estas se les ofrece atención individual); mujeres con trastornos mentales sin adherencia al tratamiento (ya que suelen ser disruptivas en los grupos o entrar en crisis). También, es requisito no entrar con niños o niñas, ya que en la Unidad Delegación no se brinda servicio de guardería y no es aconsejable que formen parte del grupo por los temas que se abordaban (Erika Rojas, comunicación personal, 30 de enero del 2019).

La metodología empleada para la atención grupal fue el Survivor Therapy Empowerment Program (STEP), el cual es un programa psicoterapéutico y psicoeducativo desarrollado por la Dra. Lenore Walker, para el trabajo con mujeres que han experimentado 


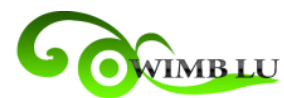

Wimblu, Rev. Estud. de Psicología UCR, 16(2) 2021 (Julio-Diciembre): 67-92 /ISSN: 1659-2107

violencia por parte de sus parejas u otras formas de violencia de género (Walker 2017). El STEP es un programa que consta de 12 unidades enfocadas en el trauma y sus efectos, específicamente en la ansiedad y sentimientos negativos; mientras que cada unidad tiene 3 componentes: psicoeducación, discusión, y desarrollo de habilidades (Walker 2017). Además, el STEP cuenta con evidencia de disminuir el malestar y la ansiedad (Walker 2017), medidas a través de 2 escalas que se detallan más adelante.

Adicionalmente, la autora de este artículo cuenta con un entrenamiento en la aplicación del STEP, ya que participó en diciembre del 2018 en una capacitación especializada impartida por la Dra. Lenore Walker, por lo que se encuentra certificada para aplicar dicha metodología.

\section{Estrategias en las intervenciones breves en la sala de espera}

Con el objetivo de brindar herramientas para el manejo del estrés y la ansiedad para las mujeres que se encontraban en la sala de espera aguardando a que sea su turno para ser atendidas, fue que se planteó crear una serie de actividades con contenido comunicativo, pedagógico o lúdico recreativo para ese espacio. Se propuso primeramente realizar observaciones de la sala de espera, para diseñar una metodología. Para estas intervenciones se planificó que fueran breves, y se impartieran a la totalidad del grupo de mujeres que se encontraban en la sala de espera, aunque la participación fue voluntaria. Esta tarea fue planteada para realizarse en momentos en que la sala de espera se encontraba llena.

\section{Resultados}

\section{Atención psicológica individual}

Se realizaron un total de 28 procesos de atención psicológica individual, de estos: alrededor del 60\% (17 mujeres) culminaron exitosamente el proceso, con un mínimo de 6 y máximo de 13 sesiones realizadas; 9 mujeres (32\%) abandonaron o renunciaron a continuar 


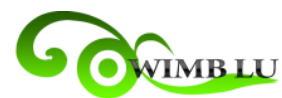

Wimblu, Rev. Estud. de Psicología UCR, 16(2) 2021 (Julio-Diciembre): 67-92 /ISSN: 1659-2107

con la atención individual; y 2 mujeres (un 7\%) fueron referidas a otra psicóloga especialista en abuso sexual. Las sesiones de atención psicológica individual se realizaban con una frecuencia variable (semanal o quincenal), dependiendo del nivel de riesgo de femicidio de cada mujer. Cada sesión tuvo una duración de 1 hora aproximadamente, aunque era posible que se extendiera para profundizar en algún tema o para realizar un cierre adecuado de la sesión.

Con relación a las manifestaciones de violencia experimentada, la más frecuente fue la violencia por parte de la pareja (21 mujeres); 4 mujeres tenían presencia de violencia por parte de pareja y abuso sexual infantil; 2 mujeres experimentaban violencia intrafamiliar; y solo una mujer reportó violencia intrafamiliar y de pareja de forma simultánea. Sobre el sexo de las parejas agresoras de estas mujeres, la mayoría eran hombres (novios, esposos, convivientes, o ex parejas); con la excepción del caso de una de las usuarias, cuya pareja también era una mujer. Con respecto al nivel de riesgo de femicidio, de las 17 usuarias que culminaron el proceso de atención individual: 2 no presentaban riesgo actual; 8 de ellas (el $47 \%$ ) estaban en bajo riesgo; 5 mujeres en riesgo moderado (29\%); y 2 calificaban como en alto riesgo de femicidio.

Sobre las referencias institucionales, se realizaron 8 en total, 4 fueron internas: 2 a asesoría legal y 2 a atención de Trabajo Social; y 4 externas a la Caja Costarricense del Seguro Social (CCSS): 2 a valoración en psiquiatría, y 2 valoraciones en psicología para la hija e hijo de usuarias del servicio. Además, a 5 usuarias se les colaboró con el escrito para solicitar las medidas de protección (el cual debía llevar al Juzgado correspondiente para que se iniciara el trámite); y en el caso de 2 mujeres fue necesario brindarles el kit de emergencia debido a las amenazas e intimidación de sus ex parejas.

Por último, se diseñó una encuesta para la evaluación del proceso de atención psicológica individual, la cual fue aplicada en la última sesión a las 17 mujeres que lo concluyeron. El 88\% de las usuarias calificó de "Excelente" el trato que recibió por parte de la practicante, que se sintió en un espacio agradable/confortable, que este espacio fue seguro y que le permitió contar su historia. El 94\% juzgó de "Excelente" la disposición de la practicante para escucharlas y atenderlas, y el no sentirse cuestionadas, ni juzgadas por sus decisiones de vida. Además, el 100\% de las usuarias en modalidad individual calificaron de "Excelente" el empleo de un vocabulario claro, que se ofreciera información de fácil acceso 


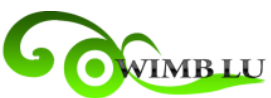

Wimblu, Rev. Estud. de Psicología UCR, 16(2) 2021 (Julio-Diciembre): 67-92 /ISSN: 1659-2107

y se brindara una atención adecuada. De igual forma, dichas usuarias consideraron que sí lograron poner en práctica conocimientos y herramientas de la atención psicológica y, además, que estos les dieron resultados positivos.

\section{Atención psicológica grupal}

Se realizó un proceso de atención psicológica grupal de 14 sesiones, de frecuencia semanal, con una participación de entre 6 a 15 mujeres por sesión, aunque la asistencia de las mujeres fue en disminución conforme avanzaba el proceso. Finalmente, 9 usuarias culminaron la atención psicológica grupal, lo cual significa que participaron del grupo de forma regular y no faltaron más de 3 veces. Además, la atención grupal se co-facilitó con otra psicóloga de la Unidad Delegación.

En cuanto a la violencia experimentada por las usuarias en atención grupal, mayormente eran víctimas de violencia por parte de la pareja, y en menor medida violencia intrafamiliar y sexual. Adicionalmente, se entregó el kit de emergencia a una usuaria en esta modalidad de atención debido a las amenazas y violencia de su ex pareja.

Por otra parte, fue necesario realizar adecuaciones a la propuesta original del STEP ${ }^{5}$, ya que este provee la información de los contenidos a abordar por cada unidad, pero no las actividades a realizar; por lo que se buscó desarrollar una intervención tipo taller, que permitiera el aprendizaje a través de actividades.

Sobre los resultados de las escalas incluidas en el STEP, primeramente, con respecto al Subjective Units of Discomfort Scores (Wolpe 1958; citado en Walker 2017) se aplicó al inicio de todas las sesiones, en las que las mujeres calificaban entre 1 a 10 su estado de ánimo de ese día. Las respuestas de las usuarias variaron entre respuestas positivas y negativas, pero se observa cierta tendencia al alza comparando las puntuaciones de las primeras sesiones con las últimas, lo que indica una disminución en los niveles de malestar de las mujeres participantes. Sobre la segunda escala incluida en el STEP, Beck Anxiety Instrument (Beck et al. 1988; citado en Walker 2017), se aplicó en 3 momentos: al inicio, a la mitad y al

\footnotetext{
${ }^{5}$ Para mayor detalle sobre la adaptación realizada al STEP se recomienda buscar el documento final de este Trabajo Final de Graduación.
} 


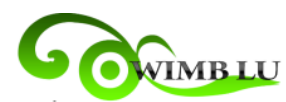

Wimblu, Rev. Estud. de Psicología UCR, 16(2) 2021 (Julio-Diciembre): 67-92 /ISSN: 1659-2107

finalizar del proceso grupal. Sin embargo, debido a que el grupo era inconstante en cuanto a la asistencia, no fue posible obtener los datos completos de estos 3 momentos; por lo que estos son insuficientes para llegar a afirmaciones concluyentes sobre los niveles de ansiedad de las participantes.

Finalmente, se aplicó una encuesta, de elaboración propia, para conocer la percepción de las usuarias sobre la atención grupal brindada, la cual fue completada por las 7 usuarias que participaron de la última sesión. Sus resultados muestran que el 100\% de las mujeres calificó como "Excelente" que las actividades: tenían instrucciones de fácil comprensión, que le permitieron aprender nuevos conocimientos, y eran adecuadas para cada tema. También, el $100 \%$ de las usuarias juzgó como "Excelente" el trato que recibió por parte de la facilitadora, la disposición que tuvo esta para escucharla y atenderla, el uso de un vocabulario claro y de fácil comprensión, y el buen dominio de los temas abordados. De igual manera, el $100 \%$ de las mujeres calificaron positivamente la puesta en práctica en su vida cotidiana de conocimientos o herramientas aprendidas durante el proceso grupal; que estos le dieron resultados positivos; que pudo compartirlos con otras personas cercanas; y que se cumplieron sus expectativas respecto al proceso de atención terapéutica grupal.

\section{Intervenciones grupales breves en la sala de espera}

Con respecto al planeamiento de las intervenciones grupales breves en la sala de espera, primeramente, se realizaron 2 observaciones en este espacio físico, en las cuales se pudo constatar que el grupo no era constante, porque las mujeres entraban y salían continuamente de acuerdo a la atención que recibían. Con base en estas observaciones, se planteó un diseño metodológico para intervenir la sala de espera, pensado para durar 20 minutos (debido a que no era posible realizar actividades extensas y elaboradas por lo inconstante y variable del grupo), que incentivara la participación activa de las usuarias y que transmitiera un mensaje positivo y esperanzador, considerando los motivos por los que las mujeres consultaban al servicio. El diseño iniciaba con un breve encuadre de 1 minuto, en el que se resaltaba que la participación era voluntaria; luego se seleccionaba una breve dinámica 


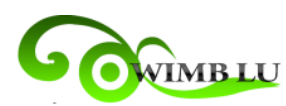

Wimblu, Rev. Estud. de Psicología UCR, 16(2) 2021 (Julio-Diciembre): 67-92 /ISSN: 1659-2107

de una variedad de actividades diseñadas, la cual se desarrollaba por 17 minutos máximo; y finalmente se realizaba una corta evaluación anónima de la intervención.

Las actividades propuestas se dividieron de acuerdo a su intencionalidad. Con intencionalidad comunicativa se propusieron 2 actividades: un “Sabías qué...?” sobre logros de mujeres costarricenses en ámbitos políticos, laborales, intelectuales y deportivos; y un verdadero o falso sobre temas de salud en general, como alimentación, el Papanicolaou y la mamografía, métodos anticonceptivos e infecciones de transmisión sexual. Con respecto a la intencionalidad pedagógica, se propusieron 2 actividades: un conversatorio sobre el autocuidado, y un análisis de canciones. Sobre las actividades con intencionalidad lúdicorecreativa se planteó realizar 2 actividades: un breve entrenamiento en respiración y relajación, y pintar mandalas.

Finalmente, se realizaron 13 intervenciones breves en la sala de espera, alcanzando a un total de 111 usuarias. Cada actividad se realizó entre 2 a 3 veces. No se pudo ejecutar la actividad de pintar mandalas porque no se contaba con superficie de apoyo para colorear.

Por último, sobre los resultados de la evaluación a estas intervenciones en la sala de espera, la mayoría de las respuestas fueron positivas. En primer lugar, a la pregunta de si se sintieron bien durante la intervención, 79 usuarias (72\%) respondieron que estaban "Totalmente de acuerdo"; mientras 23 usuarias (21\%) contestaron que estaban "De acuerdo", y 7 respondieron de forma neutral. La segunda pregunta, de si aprendieron algo nuevo o útil, 70 usuarias (64\%) lo calificó como "Totalmente de acuerdo", 37 participantes (34\%) respondieron con un "De acuerdo", y 2 mujeres contestaron no estar ni de acuerdo ni en desacuerdo. Sobre si la actividad les pareció interesante, 87 usuarias (80\%) respondieron estar "Totalmente de acuerdo"; 21 usuarias estuvieron "De acuerdo"; y solo 1 participante respondió neutralmente a esta cuestión. No se registraron respuesta en desacuerdo a las 3 preguntas mencionadas.

\section{Otras tareas solicitadas por la institución}

\section{Atención en rol}

Esta se refiere a la atención que reciben las mujeres que visitan por primera vez la institución, la cual tiene la función de determinar el motivo de consulta, valorar el nivel de 


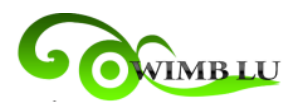

Wimblu, Rev. Estud. de Psicología UCR, 16(2) 2021 (Julio-Diciembre): 67-92 /ISSN: 1659-2107

riesgo de femicidio, identificar la manifestación de violencia experimentada, y realizar las referencias o contra-referencias necesarias para la atención adecuada de cada mujer. De esa forma, se atendieron en rol 64 usuarias durante el desarrollo de esta práctica dirigida. Sobre el nivel de riesgo de femicidio de esas usuarias, se identificó que la mayoría de ellas (21 usuarias) estaban en riesgo moderado; 20 mujeres calificaron como bajo riesgo; mientras que en alto riesgo estaban 15 de ellas; y 8 usuarias no presentaban riesgo de femicidio actual.

Finalmente, se realizaron 60 referencias a estas usuarias: 54 fueron internas (26 a atención psicológica, 14 a atención social, 6 a asesoría legal y 8 a atención grupal) y 6 externas (a una Oficina de la Mujer (OFIM), Casa de Derechos de la Municipalidad de Desamparados, valoración en psiquiatría de la Caja Costarricense del Seguro Social, y a Emergencias del Hospital Rafael Ángel Calderón Guardia).

\section{Talleres psicoeducativos}

Se colaboró en la planificación e implementación de 4 talleres psicoeducativos: 2 sobre derechos humanos de las mujeres, y 2 sobre derechos sexuales y reproductivos. Cada taller era de una única sesión, con una duración de 3 a 4 horas, y se llevaron a cabo con grupos de apoyo ya existentes en el servicio. Estos fueron co-facilitados con otra psicóloga de la Unidad Delegación. Finalmente, participaron 79 usuarias, con grupos conformados por un máximo de 23 mujeres y un mínimo de 12 usuarias.

\section{Entrevista a una víctima de trata de personas}

Cuando en la Unidad Delegación se detecta una posible víctima de trata de mujeres, de acuerdo con la Ley contra la Trata de Personas y Creación de la Coalición Nacional contra el Tráfico Ilícito de Migrantes y la Trata de Personas (Ley 9095/2012), se debe de realizar una entrevista en profundidad sobre los elementos constitutivos del delito, con la que se realiza un informe para solicitar ante el Equipo de Respuesta Inmediata de la Coalición Nacional contra el Tráfico Ilícito de Migrantes y la Trata de Personas (CONATT) su acreditación como víctima de este delito; si la persona es acreditada como tal, entra a una 


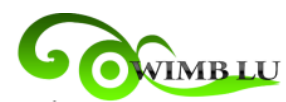

Wimblu, Rev. Estud. de Psicología UCR, 16(2) 2021 (Julio-Diciembre): 67-92 /ISSN: 1659-2107

estrategia estatal para su atención integral. Siguiendo este procedimiento, se participó como traductora de inglés al español en la entrevista de una usuaria de origen estadounidense, detectada como víctima de trata de personas con fines de explotación sexual. Esta se dividió en 2 días debido a la gran cantidad de información que se debió recabar.

\section{Análisis y discusión de resultados}

\section{Vulnerabilidad de las mujeres}

Fue posible identificar algunos aspectos que colocaban a las mujeres en una condición de vulnerabilidad. Primeramente, el poco acceso a información o el desconocimiento (total o parcial) sobre derechos humanos de las mujeres, no reconocimiento de la violencia y naturalización de la misma. Esto se puede entender debido a la aceptación social e implícita de la violencia en contra de las mujeres, puesto que ha sido invisibilizada históricamente y justificada socialmente (Mesa 2014). De esta forma, como se enseña, a nivel de sociedad, que la violencia en contra de las mujeres es natural o "normal", las usuarias lo han llegado a aprender. Por este motivo, es que es un principio básico en la intervención con estas mujeres visibilizar y desnaturalizar la violencia, ya que esta provoca una habituación en las víctimas, llevando a que no perciban subjetivamente el maltrato de sus parejas de esa forma (Deza 2016). Adicionalmente, es fundamental incorporar la perspectiva de género en las atenciones, para que las mujeres puedan recuperar el control de sus vidas (Alencar y Cantera 2013).

Otro aspecto importante a destacar es que las condiciones materiales de las mujeres las colocaban en una posición de vulnerabilidad. Como se mencionó anteriormente, la mayoría de usuarias no terminaron la primaria o secundaria, y estaban desempleadas, lo que las llevaba a trabajos con salario mínimo o al sector informal; también, muchas de ellas se encontraban en condición de pobreza. Así, el bajo nivel socioeconómico y el desempleo tienen un rol decisivo en la violencia, debido a que remarcan las desigualdades de poder en relaciones de pareja, en las que las mujeres se encuentran en una posición de subordinación en cuanto a educación, ingresos y ocupación con respecto a los hombres (Vives 2011). 
Asimismo, fue necesario considerar el concepto de interseccionalidad, el cual refiere al "sistema complejo de estructuras de opresión que son múltiples y simultáneas" (Crenshaw 1995; citada en Muñoz 2011, 10), por lo que categorías como clase social, condición migratoria, etnia, edad, orientación sexual, entre otras, se cruzan con la opresión de género, creando formas particulares de desigualdad y de vulnerabilidad para las mujeres (Sagot 2017). Este análisis fue esencial en la atención de las usuarias, debido a que el riesgo no era igual para todas, ya que la peligrosidad y severidad de la violencia y las opciones para salvaguardar sus vidas y sobrevivir variaba dependiendo de la configuración de estas opresiones (Sagot 2017).

Por otra parte, fue posible identificar cómo las usuarias postergaban sus propias necesidades, lo que se explica debido a que parte de la identidad femenina hegemónica es estar al servicio de las otras personas que les rodean; lo que además impacta la capacidad de decisión de las mujeres (Mesa 2014). Además, las usuarias aprendieron de relaciones de pareja a partir de sus padres y madres (y familiares en general), relatando conductas manipuladoras y violentas, de obediencia al padre, sin cuestionar o discutir, entre otras. Al respecto, las relaciones familiares tradicionalmente se fundamentan en valores patriarcales, lo que construye "(...) una familia de estructura vertical, donde el poder se concentra en el llamado «jefe de familia», que se encuentra jerárquicamente por encima de la esposa y las hijas e hijos" (Mesa 2014, 24). De esta forma, se establece una noción de obediencia dentro de la familia, en la que se hace la voluntad del hombre, instaurando relaciones de dominiosumisión, especialmente en relaciones de pareja entre hombres y mujeres (Álvarez et al. 2016).

Sobre el impacto de la violencia en la salud mental de las mujeres, se identificaron mujeres con trastornos de ansiedad, depresión, bipolaridad, esquizofrenia, internamientos psiquiátricos, ideación e intentos de suicidio; y algunas eran medicadas con psicotrópicos, como fluoxetina, amitriptilina, imipramina, diazepam, entre otros. Con respecto a lo anterior, la cuestión está en que habitualmente las personas profesionales en salud mental entienden que es una supuesta psicopatología subyacente la causa de la vivencia de violencia, en vez de entender la sintomatología como una respuesta a la situación de abuso (Lorente 1999, Villavicencio y Sebastián 1999, Corsi 2003; citados en Deza 2016). Asimismo, la prescripción de psicofármacos refuerza la noción de que las mujeres son las culpables de su 


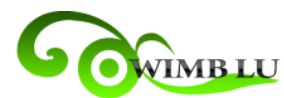

Wimblu, Rev. Estud. de Psicología UCR, 16(2) 2021 (Julio-Diciembre): 67-92 /ISSN: 1659-2107

situación, y psicopatologizan los síntomas de las mismas, ya que las coloca como enfermas (Cabruja 2005; citado en Alencar y Cantera 2013). Además, se señalan los prejuicios basados en el género cuando se prescriben medicamentos psicotrópicos, ya que es muy probable que a las mujeres se los receten (Pereira 2018).

\section{Dinámica de violencia}

Con respecto a los factores que mantenían a las usuarias en la relación de abuso, se podría iniciar mencionando la dependencia económica de las mujeres hacia la persona agresora. Al respecto, la escasez de recursos económicos se vincula con permanecer en la relación de violencia (Alencar y Cantera 2013). Además, como se ha mencionado, la dependencia económica propicia la construcción de relaciones de pareja desiguales, en las que el hombre tiene el poder económico y el poder en el hogar (Mesa 2014).

Otro punto a considerar fueron las ideas del amor romántico, las cuales enseñan que el amor es una entrega total, que el amor lo puede todo, sus parejas se volvían el centro de su existencia, perdonándolos y justificándolos todo "por amor", convirtiéndose en uno de sus proyectos elementales de vida, y renunciando a sus propios intereses (Álvarez et al. 2016).

Asimismo, el ciclo de violencia, conformado por 3 fases y caracterizado porque en las relaciones de violencia existan momentos de tensión, agresión, cariño y calma, puede explicar por qué las mujeres permanecían en la relación de abuso, ya que en la tercera fase (luego de ocurrido el episodio de violencia) las personas agresoras suelen aceptar sus errores, disculparse y prometer cambios, llegando a utilizar estrategias de manipulación afectiva, como regalos, caricias, promesas, entre otros (Álvarez et al. 2016); y es justo eso lo que proporciona un reforzamiento positivo para mantenerse en la relación (Walker 2017).

Otro aspecto evidenciado para continuar en la relación fue la presión familiar y social. La "trampa del sacrificio" (Mesa 2014, 24) es entendida como la obligación de las mujeres de conservar la unidad familiar, priorizando los intereses de las otras personas antes que los propios. Además, a nivel familiar se suele responsabilizar de la violencia a la víctima, y no al abusador, lo que aumenta la revictimización y desincentiva denunciar a alguien del núcleo familiar (Mesa 2014). 


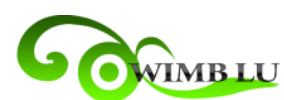

Wimblu, Rev. Estud. de Psicología UCR, 16(2) 2021 (Julio-Diciembre): 67-92 /ISSN: 1659-2107

Por otra parte, algunas usuarias señalaban que "por el bien de sus hijas/os" se quedaba en la relación de abuso, ya que expresaban temor de que su descendencia no tuviera figura paterna; aunque, contradictoriamente, en la mayoría de los casos esa figura era solo proveedora económica, y no un apoyo a nivel emocional, ni colaboraban en los estudios y crianza de sus hijas/os (como se menciona más adelante).

Otra cuestión a considerar fue la religión, ya que algunas usuarias mencionaban que el matrimonio era sagrado, por lo que solicitar el divorcio a sus esposos no era una opción. Al respecto, la mayoría de religiones mantienen a las mujeres en un rol de sumisión, sostenida por una jerarquía masculina e instaura el sometimiento y control de las mujeres desde sus mitos fundantes (Mesa 2014).

Por último, en el caso de la única mujer lesbiana en atención individual, permanecía en la relación de abuso por el temor de perder los bienes gananciales adquiridos durante los años de relación, ya que esta intervención se realizó antes del 26 de mayo del 2020, cuando las parejas del mismo sexo aún no contaban con los mismos derechos de las parejas heterosexuales.

\section{Crianza de sus hijas/os}

Otro tema que fue frecuentemente abordado por las mujeres fue la relación con sus hijas/os. Las usuarias identificaban el impacto que tenía en sus hijas/os el haber sido testigas o víctimas directas de violencia, ya que presentaban comportamientos desafiantes, actitudes violentas, afectación en la confianza en sí misma/o, bajo rendimiento en la escuela o el colegio, aumento en la ansiedad, ideación e intentos de suicidio, entre otros.

Por otra parte, la exposición a la violencia influye en los procesos de socialización y apropiación de roles de género tradicionales de las niñas y niños. Este aprendizaje intergeneracional de la violencia se manifiesta de diferentes formas según el género: las niñas víctimas tienen mayores posibilidades de experimentar violencia en su adultez, mientras que los niños víctimas tienden a ser victimarios en mayor medida (Vives 2011).

Asimismo, las usuarias reportaban el poco o nulo apoyo de los padres en la crianza de sus hijas/os, algunos hombres eran solamente proveedores económicos, otros ni pagaban 


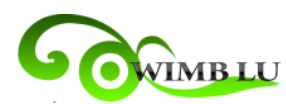

Wimblu, Rev. Estud. de Psicología UCR, 16(2) 2021 (Julio-Diciembre): 67-92 /ISSN: 1659-2107

la pensión alimentaria, por lo que las usuarias solicitaban continuamente órdenes de apremio corporal o, llegar al punto, de buscar a su ex pareja a algún lugar público con la policía para que se pueda hacer efectivo el mismo. Estas dificultades económicas para hacerse cargo de sus hijas/os afectaba la salud mental de las mujeres, les causaba estrés, desánimo, desgaste y desesperación.

Por otra parte, algunas usuarias tenían procesos con el Patronato Nacional de la Infancia (PANI), debido a que esta institución consideró que se estaban irrespetando los derechos de la niñez y la adolescencia por razones como: abuso emocional y físico (lo que incluye el castigo físico); negligencia; o consumo de sustancias psicoactivas por parte de la madre o el padre.

Un aspecto recurrente identificado en la narrativa expresada por algunas usuarias atendidas fueron las malas experiencias en el trato recibido por parte de las personas profesionales que las atendían en el PANI (en su mayoría eran de Psicología y Trabajo Social), lo que incluía actitudes que juzgaban a las mujeres por permanecer con la pareja agresora, las culpabilizan por el daño que la violencia causó en sus hijas/os, no consideraban la situación de violencia, la minimizaban o del todo no les creían, favoreciendo al progenitor agresor. Lo anterior constituye un problema en el accionar institucional, debido a que un acercamiento profesional sin perspectiva de género es un recurso obstaculizador de la reducción de violencia por parte de la pareja (Alencar y Cantera 2013). Además, cuando hay violencia por parte de la pareja no son aconsejadas las intervenciones basadas en la mediación familiar, dado que esta posiciona a las mujeres en desventaja (Romero 2010; citada en Alencar y Cantera 2013). Adicionalmente, un posicionamiento claro en contra de la violencia por parte de la persona profesional es un principio básico en la intervención con mujeres víctimas de violencia (Deza 2016); mientras que un accionar profesional de neutralidad, que obvie las vivencias de violencia de las mujeres, reproduce una lógica de dominación que encubre y normaliza la violencia (Corsi 2003; citado en Deza 2016) 


\section{Procesos legales e impunidad}

Un aspecto vivenciado por muchas de las usuarias atendidas era que se encontraban llevando algún proceso legal. Al respecto, quedó en evidencia la necesidad de las mujeres de asesoramiento y representación legal, ya que muchas de ellas expresaban desconocimiento sobre estos procesos y la falta de recursos económicos para cubrir los gastos legales, ante lo cual se realizaron referencias a las abogadas del servicio y a Consultorios Jurídicos de la Universidad de Costa Rica (aunque este tenía una capacidad limitada, por lo que se debía contar con el visto bueno de la Jefatura de la Unidad Delegación para realizar referencias a este servicio).

Asimismo, esos procesos legales en marcha llegaban a afectar la salud mental de las usuarias, quienes presentaban cansancio mental y anímico, se sentían abrumadas, con enojo, desesperación, impaciencia, estrés e incertidumbre. Era frecuente que tuvieran dificultades con ciertos procesos específicos, como con la notificación de las medidas de protección (el cual es un requisito para que entren en vigencia las mismas), debido a que la policía no encontraba a la persona agresora en su domicilio, o las personas que vivían con él lo protegían. Similarmente, era bastante común que los hombres no pagaran la pensión alimentaria, por lo que las mujeres optaban por el apremio corporal contra la persona morosa, aunque en ocasiones no era efectivo debido a que la persona deudora no se encontraba en su domicilio.

No obstante, en algunos casos, las dificultades con procesos legales eran debido a la inoperancia de las fuerzas policiales y judiciales, quienes eran cómplices al no realizar su trabajo adecuadamente. $\mathrm{Al}$ respecto, las personas funcionarias de justicia a menudo realizan su trabajo desde preconcepciones tradicionales, discriminatorias y sexistas, lo que entorpece en gran medida el acceso a la justicia para las mujeres víctimas de violencia (Sagot 2008). Además, cuando las estructuras sociales e institucionales no protegen la integridad de las víctimas se incurre en violencia estructural, lo que provoca impunidad y revictimización (Pereira 2018) 


\section{Atención grupal}

Un primer aspecto a destacar del proceso de atención psicológica grupal fue que posibilitó que las mujeres compartieran experiencias con otras integrantes del grupo, las cuales se relacionaban con sobrevivencia de la violencia, conflictos personales y familiares, desafíos en la crianza de sus hijas/os, entre otras. De esa forma, el intercambio de vivencias entre las participantes facilita que dimensionen la violencia experimentada (Deza 2016), y promueve la identificación y validación de sus experiencias a través del discurso de otras compañeras (Ramírez et al. 2005; citadas en Alencar y Cantera 2013).

Además, la atención grupal favoreció la construcción de redes de apoyo y amistad entre las usuarias. Así, compartir con otras mujeres en una situación similar proporciona apoyo emocional, disminuye el aislamiento social en el que se pueden encontrar mujeres víctimas y/o sobrevivientes de violencia (Deza 2016), y posibilita el aprendizaje de las estrategias que utilizaron sus compañeras para afrontar y terminar la relación de abuso (Hughes y Jones 2000; citadas en Deza 2016).

Otro aspecto importante a destacar es que la atención grupal facilitó la adquisición de habilidades y conocimientos por parte de las usuarias, por ejemplo, utilizaban técnicas como el tiempo fuera, la respiración, llevar un registro de su estado de ánimo y pensamientos, entre otros, empleaban conceptos como ciclo de violencia, patriarcado, roles de género y cuestionaban los estereotipos de género; lo que facilita la identificación y análisis de los factores estructurales y culturales que legitiman la violencia (Albarracín et al. 2007; RocaCortés et al. 2007; citadas en Alencar y Cantera 2013).

Aunado a lo anterior, una característica de las intervenciones grupales es la recuperación del daño vinculado con la violencia experimentada (Deza 2016), lo que fue posible identificar en el relato de las mismas usuarias, quienes reportaban una disminución en lo que ellas mismas distinguían como malestar, ansiedad, estrés y depresión, y un aumento en su propia confianza y satisfacción con la vida. 


\section{Conclusiones}

En esta inserción profesionalizante se desarrollaron procesos de atención psicológica en modalidades individuales y grupales en la Unidad Delegación de la Mujer del INAMU con mujeres víctimas y/o sobrevivientes de violencia por parte de la pareja, intrafamiliar, sexual, trata de personas, entre otros. Estas intervenciones posibilitaron cambios en sus vidas, ya que pudieron identificar su situación como de violencia, aprendieron sobre estrategias para salvaguardar su vida y la de sus hijas/os, a desnaturalizar la violencia, cuestionar roles y estereotipos de género, e identificar características de una relación de pareja saludable. También, permitieron que las mujeres tuvieran un mejor manejo de sus emociones, se fijaran metas personales, y promovió el procesamiento de las experiencias de violencia de las usuarias, favoreciendo la recuperación emocional. Además, durante la atención psicológica individual y la grupal, se construyó una alianza terapéutica entre la autora y las usuarias, lo que promovió que las mujeres entraran en confianza, se permitieran relatar sus vivencias de violencia (para algunas de las usuarias fue la primera vez que hablaban con alguien de sus experiencias de abuso) y posibilitaron el proceso de cambio. De esta manera, fue posible fortalecer la respuesta a la problemática de la violencia contra las mujeres en la Unidad Delegación; por lo que se considera que se han cumplido satisfactoriamente con los objetivos fijados para este trabajo.

Asimismo, en el desarrollo de esta práctica dirigida se identificó que efectivamente la violencia en contra de las mujeres lesiona el ejercicio de sus derechos humanos y su pleno desarrollo. A nivel de salud física, las usuarias reportaban desde lesiones leves, hasta daños mayores que requirieron intervención médica, incluso intentos de femicidio; pérdida de peso, presión sanguínea alterada, hemograma alterado, alergias en la piel, crisis de asma, fibromialgia, entre otros. En cuanto a la salud mental, se identificó ansiedad, insomnio, baja autoestima, dificultad para tomar decisiones propias, hipersensibilidad, entre otros. También, se constataron limitaciones para ejercer sus derechos sexuales y reproductivos, contagio de infecciones de transmisión sexual, embarazos no deseados, abortos espontáneos, entre otros.

Igualmente, con base en este acercamiento a la violencia en contra de las mujeres, se evidenció la aceptación y justificación a nivel social de la misma, ya que esta es una 
problemática que permea toda la sociedad, por lo que los esfuerzos para abordarla deben de direccionarse en todos los niveles, desde lo más personal, hasta lo más macrosocial.

Por otra parte, se cuestiona por qué existe personal limitado destinado a su atención, pese a que esta es una problemática tan extendida en toda la sociedad costarricense, a la alta demanda evidenciada en esta práctica dirigida, y la gran carga laboral para las funcionarias de la Unidad Delegación.

Por último, fue posible identificar, a partir de las experiencias de las usuarias sobre ciertos acercamientos profesionales poco éticos, cómo la Psicología se puede posicionar como una ciencia cargada de poder, reproduciendo un discurso y prácticas conservadoras; pero también para facilitar intervenciones emancipadoras. Por lo anterior, es que es tan importante incorporar la perspectiva de género en normativas y prácticas institucionales; por lo que es responsabilidad de esas instituciones encargadas de abordar esta problemática de actuar de acuerdo a las convenciones internacionales de derechos humanos de las mujeres, con las que se comprometió el Estado Costarricense, y a capacitar al personal a través de procesos de sensibilización, para que realicen su trabajo lejos de preconcepciones tradicionales y prejuicios sexistas, clasistas, xenofóbicos, entre otros.

\section{Referencias}

Alencar, Roberta y Leonor Cantera. 2013. «Intervención en violencia de género en la pareja: el papel de los recursos institucionales». Athenea Digital 13 (3): 75-100. doi: 10.5565/rev/athenead/v13n3.1058

Álvarez, Mariángeles, Ana Sánchez, Pepa Bojó, Karmele Zelaiaran, Asier Aseguinolaza, Teresa Azanza y Silvia Caballero. 2016. Manual de atención psicológica a víctimas de maltrato machista. España: Colegio Oficial de la Psicología de Gipuzkoa.

Batres, Gioconda. 2013. «El abandono terapéutico de las víctimas de violencia sexual: Un problema latinoamericano. Propuestas y metodología de la terapia trifásica género sensitiva». En Género, feminismos y diversidades, editado por el Instituto de Estudios de la Mujer de la Universidad Nacional, 43-52. Heredia, Costa Rica: Instituto de Estudios de la Mujer. 
Wimblu, Rev. Estud. de Psicología UCR, 16(2) 2021 (Julio-Diciembre): 67-92 /ISSN: 1659-2107

Batres, Gioconda. 2009. «La terapia género sensitiva con víctimas y perpetradores de la violencia sexual: Un aporte latinoamericano». Acceso el 10 de febrero del 2019. http://www.giocondabatres.com/modules/news/print.php?storyid=2

Deza, Sabina. 2016. «Modelo de atención para mujeres víctimas de violencia familiar albergadas en hogares de refugio temporal. HRT». Av.psicol. 24 (1): 85-102. Acceso $\begin{array}{lllll} & 30 & \text { de } & \text { abril } & \text { del }\end{array}$ http://www.unife.edu.pe/publicaciones/revistas/psicologia/2016_1/Sabina.Deza.pdf

García, Paloma y Carmen Borja. 2014. «El EBDH: una nueva mirada al desarrollo y a los derechos humanos». En Derechos Humanos y Desarrollo. El Enfoque Basado en Derechos Humanos en la Cooperación al Desarrollo - EBDH, editado por Institut de Drets Humans de Catalunya, 11-44. Barcelona, España: Institut de Drets Humans de Catalunya.

INAMU (Instituto Nacional de las Mujeres). 2020. «Información estadística del total de atenciones individuales de la Delegación de la Mujer 2016-2019». Datos inéditos sin publicar.

INAMU (Instituto Nacional de las Mujeres). 2017a. Política Nacional para la Atención y la Prevención de la Violencia contra las Mujeres de todas las Edades Costa Rica 2017 - 2032. San José, Costa Rica: Editorial EUNED.

INAMU (Instituto Nacional de las Mujeres). 2017b. Protocolo interinstitucional de intervención. Valoración y administración del riesgo en situaciones de violencia contra las mujeres en relaciones de pareja. San José, Costa Rica: Editorial EUNED.

INAMU (Instituto Nacional de las Mujeres). 2010. Plan Estratégico 2011 - 2014. San José, Costa Rica: Instituto Nacional de las Mujeres.

Mesa, Sylvia. 2014. «Implicaciones de la justificación social de la violencia contra las mujeres». En La Violencia en Costa Rica en los albores del nuevo milenio, editado por Mario Sáenz y Mirta González, 203-223. San José, Costa Rica: Guayacán.

Ministerio de Desarrollo Social. 2010. Protocolo de atención para los servicios especializados de atención a mujeres en situación de violencia doméstica del Instituto Nacional de las Mujeres MIDES. Acceso el 30 de abril del 2019. http://dspace.mides.gub.uy:8080/xmlui/bitstream/handle/123456789/719/1300protocoloserviciosinmujeres.pdf? sequence $=1 \&$ isAllowed $=\mathrm{y}$ 
Wimblu, Rev. Estud. de Psicología UCR, 16(2) 2021 (Julio-Diciembre): 67-92 /ISSN: 1659-2107

Morales, Carla y Vera Aguilar. 2016. Caracterización de la oferta y demanda de servicios de violencia contra las mujeres, a nivel nacional, regional y local. Documento Final. Costa Rica: Centro de estudios democráticos de América Latina.

Muñoz, Patricia. 2011. Violencias Interseccionales. Debates Feministas y Marcos Teóricos en el tema de Pobreza y Violencia contra las Mujeres en Latinoamérica. Tegucigalpa, Honduras: Central America Women's Network (CAWN).

Observatorio de Violencia de Género contra las Mujeres y Acceso a la Justicia. 2020a. «Violencia doméstica». Acceso el 17 de febrero del 2020. https://observatoriodegenero.poder-judicial.go.cr/index.php/soy-especialista-ybusco/estadisticas/violencia-domestica

Observatorio de Violencia de Género contra las Mujeres y Acceso a la Justicia. 2020b. «Ley de Penalización de la Violencia contra la Mujer». Acceso el 17 de febrero del 2020. https://observatoriodegenero.poder-judicial.go.cr/index.php/soy-especialista-ybusco/estadisticas/ley-de-penalizacion-de-la-violencia-contra-la-mujer

Observatorio de Violencia de Género contra las Mujeres y Acceso a la Justicia. 2020c. «Femicidio». Acceso el 17 de febrero del 2020. https://observatoriodegenero.poderjudicial.go.cr/index.php/soy-especialista-y-busco/estadisticas/femicidio

Organización Internacional para las Migraciones. 2007. Guía de intervención psicosocial para la asistencia directa con personas víctimas de trata. San José, Costa Rica: Oficina Regional para Centroamérica y México.

OMS (Organización Mundial de la Salud). 2013. Global and regional estimates of violence against women: prevalence and health effects of intimate partner violence and nonpartner sexual violence. Italia: OMS.

OPS (Organización Panamericana de la Salud). 2016. Atención de salud para las mujeres que han sufrido violencia de pareja o violencia sexual. Manual clínico. Washington, D.C.: OPS.

OPS y CDC (Organización Panamericana de la Salud y Centros para el Control y la Prevención de Enfermedades de los Estados Unidos). 2014. Violencia contra las mujeres en América Latina y el Caribe: Análisis comparativo de datos poblacionales de 12 países. Washington, DC: OPS 
Wimblu, Rev. Estud. de Psicología UCR, 16(2) 2021 (Julio-Diciembre): 67-92 /ISSN: 1659-2107

Pereira, Sandra. 2018. «Salud, mujeres y violencia doméstica». Revista Judicial (123): 2539. Acceso el 8 de julio del 2020. https://www.corteidh.or.cr/tablas/r38554.pdf

Rodríguez, Yuriria, Cecilia Robledo y Teresa Pedroso. 2010. Guía para la Incorporación de la Perspectiva de Género en Programas de Salud. México: Centro Nacional de Equidad de Género y Salud Reproductiva.

Sagot, Monserrat. 2017. «¿Un mundo sin femicidios? Las propuestas del feminismo para erradicar la violencia contra las mujeres». En Feminismos, pensamiento crítico y propuestas alternativas en América Latina, coordinado por Monserrat Sagot, 61-78. Buenos Aires: CLACSO.

Sagot, Monserrat. 2008. «Estrategias para enfrentar la violencia contra las mujeres: reflexiones feministas desde América Latina». Athenea Digital (14): 215-228. Acceso el 8 de julio del 2020. http://mujeresdeguatemala.org/wpcontent/uploads/2014/06/Estrategias-para-enfrentar-la-violencia-cjontra-lasmujeres.pdf

Sagot, Monserrat y Laura Guzmán. 2004. Encuesta Nacional de violencia contra las mujeres. $\begin{array}{llllll}\text { Acceso } & \text { el } & 31 & \text { de } & \text { enero }\end{array}$ http://repositorio.ciem.ucr.ac.cr/bitstream/123456789/86/1/RCIEM070.pdf

Vives, Carmen. 2011. «Un modelo ecológico integrado para comprender la violencia contra las mujeres». Feminismo/s (18): 291-299. doi: 10.14198/fem.2011.18.16

Walker, Lenore. 2017. The Battered Woman Syndrome (Fourth Edition). New York: Springer Publishing Company. EBSCO (AN 1398434).

\section{Leyes}

Ley 6968/1984, 2 de octubre, Convención sobre la eliminación de todas las formas de discriminación contra la mujer (Diario Oficial La Gaceta $N^{\circ} 8$ del 11 de enero de 1985).

Ley 7499/1995, 2 de mayo, Convención Interamericana para prevenir, sancionar y erradicar la violencia contra la mujer "Convención Belem Do Pará" (Diario Oficial La Gaceta $\mathrm{N}^{\circ} 123$ del 28 de junio de 1995).

Ley 7586/1996, 10 de abril, Ley contra la Violencia Doméstica (Diario Oficial La Gaceta N ${ }^{\circ}$ 83 del 2 de mayo de 1996). 


\section{ranum}

Wimblu, Rev. Estud. de Psicología UCR, 16(2) 2021 (Julio-Diciembre): 67-92 /ISSN: 1659-2107

Ley 8589/2007, 25 de abril, Ley de Penalización de la Violencia Contra las Mujeres (Diario Oficial La Gaceta $\mathrm{N}^{\circ} 103$ del 30 de mayo de 2007).

Ley 9095/2012, 26 de octubre, Ley contra la Trata de Personas y Creación de la Coalición Nacional contra el Tráfico Ilícito de Migrantes y la Trata de Personas (CONATT) (Diario Oficial La Gaceta $N^{\circ} 28$ del 8 de febrero de 2013). 